Modern Physics Letters A,

(C) World Scientific Publishing Company

\title{
LORENTZ MULTIPLET STRUCTURE OF BARYON SPECTRA AND RELATIVISTIC DESCRIPTION
}

\author{
M. KIRCHBACH * \\ Institut für Kernphysik, \\ J. Gutenberg Universität Mainz, D-55099 Mainz, Germany
}

\begin{abstract}
The pole positions of the various baryon resonances are known to reveal well-pronounced clustering, so-called Höhler clusters. For nonstrange baryons the Höhler clusters are shown to be identical to Lorentz multiplets of the type $\{j, j\} \otimes[\{1 / 2,0\} \oplus\{0,1 / 2\}]$ with $j$ being a half-integer. For the $\Lambda$ hyperons below $1800 \mathrm{MeV}$ these clusters are shown to be of the type $\{1,0\} \oplus\{0,1\} \otimes[\{1 / 2,0\} \oplus\{0,1 / 2\}]$ while above $1800 \mathrm{MeV}$ they are parity duplicated $\{J, 0\} \oplus\{0, J\}$ higher-spin (Weinberg-Ahluwalia) states. Therefore, for $\Lambda$ hyperons the restoration of chiral symmetry takes place above $1800 \mathrm{MeV}$. Finally, it is demonstrated that the description of spin-3/2 particles in terms of a 2nd rank antisymmetric Lorentz tensor with Dirac spinor components does not contain any offshell parameters and avoids the main difficulties of the Rarita-Schwinger description based upon a 4 -vector with Dirac spinor components.
\end{abstract}

\section{Introduction}

A recent analysis of the pole positions of various baryon resonances $\left(N^{*}\right)$ with masses below $\sim 2500 \mathrm{MeV}$ performed by Höhler et al. reveals a well-pronounced clustering. This is quite a surprising result in that so far it was not anticipated by any model or theory 6 Subsequently, these baryon clusters will be referred to as 'Höhler clusters' according to a suggestion of Nefkens. In conjunction with this observation, the symmetry of all reported nonstrange baryon excitations with masses below $2500 \mathrm{MeV}$ was re-analyzed 3 and shown to be governed by $\mathrm{SL}(2, \mathrm{C}) \otimes \mathrm{SU}(2)_{I}$. As long as the group $\mathrm{SL}(2, \mathrm{C})$ is the universal covering of the Lorentz group, the new classification scheme for baryons is determined by $\mathrm{O}(1,3) \otimes \mathrm{SU}(2)_{I}$ rather than by $\mathrm{O}(3) \otimes \mathrm{SU}(6)$ which constantly has been used since the invention of the naive three flavor quark model till now.

The $\mathrm{O}(1,3) \otimes \mathrm{SU}(2)_{I}$ symmetry indicates that the spin-orbital correlation between quarks is much stronger than the spin-flavor one. For that reason, the production of relativistic multiplets of the type $\{j, j\} \otimes[\{1 / 2,0\} \oplus\{0,1 / 2\}]$ (with $2 j+1$ even), and thereby of resonance clusters, seems to be favored in nature over that of isolated $\{J, 0\} \oplus\{0, J\}$ higher-spin states. It is one of the purposes of the present investigation to point out an equivalence between the Lorentz multiplets and the Höhler clusters.

The existence of $\mathrm{O}(1,3) \otimes \mathrm{SU}(2)_{I}$ symmetry for baryons encountered in Ref. [3 might be quite advantageous in solving the problem of the relativistic description of

\footnotetext{
*E-mail: mariana@kph.uni-mainz.de
} 
their spectra. ${ }^{a}$ Indeed, as long as the projection operator onto each given representation of the Lorentz group is a well defined mathematical object, one can immediately write down the relativistic equation of motion describing the resonance cluster as a whole and obtain the corresponding propagator. In evaluating Feynman graphs, describing physical processes such as meson production off protons, it appears quite reasonable, therefore, to use a propagating Höhler cluster as an intermediate state, rather than an isolated higher-spin state.

Lorentz multiplets of the type observed in the baryon spectra were first considered systematically by Weinberg in connection with relativistic equations of motion for particles with any spin. 1 There, the representations $\{J, 0\} \oplus\{0, J\}$ of the Lorentz group have been mapped onto multiplets of the type $\left\{\frac{1}{2} l, \frac{1}{2} l\right\} \otimes[\{1 / 2,0\} \oplus\{0,1 / 2\}]$ with integer $l$. Such multiplets neccessarily contain $(2 l+1)$ states with spins $J$ ranging from $J=1 / 2$ to $J=l+1 / 2$. Both representations mentioned above are described in terms of totally symmetric $2 l+2-$ rank multispinors. The isolated field of maximal spin is then obtained after eliminating all the redundant fields with lower spins by means of a suitably chosen set of subsidiary conditions. It is well known that all equations of motion for particles with arbitrary spins based upon $\left\{\frac{1}{2} l, \frac{1}{2} l\right\} \otimes[\{1 / 2,0\} \oplus\{0,1 / 2\}]$ representations possess a pathological property. When placed in an electromagnetic filed, the higher-spin field $\left(N^{*}\right)$ can propagate with superluminal velocity and violate causality 5 In addition, arbitrary parameters arise both in the propagator and the $N N^{*} \gamma$ vertex when the particle is treated off-mass shell. $\mathrm{V}$ Recent progress in the description of the $\{J, 0\} \oplus\{0, J\}$ states avoiding the first difficulty was achieved by Ahluwalia et al. 8 where the states considered were treated as multicomponent bi-vectors rather than as $(2 J+1)$-rank multispinors. The covariant behavior of these states with respect to Lorentz transformations was then ensured by construction in terms of the explicit representation of the boost operation within the corresponding $2(2 J+1)$ dimensional spaces. In the following, isolated $\{J, 0\} \oplus\{0, J\}$ states will sometimes be referred to as Weinberg-Ahluwalia states to distinguish them from Höhler clusters.

The most popular equation of motion for a particle with higher spin is the one built upon the $\{1 / 2,1 / 2\} \otimes[\{1 / 2,0\} \oplus\{0,1 / 2\}]$ representation, known as the RaritaSchwinger (RS) equation 1 It describes a spin- $3 / 2$ particle together with two further spin $-1 / 2$ fields (that need be eliminated by a set of suitably constructed subsidiary conditions) in terms a Lorentz vector with Dirac spinor components. Here we compare the RS equation to the one based on the $[\{1,0\} \oplus\{0,1\}] \otimes[\{1 / 2,0\} \oplus$ $\{0,1 / 2\}]$ representation space that describes a two-resonance cluster with spins $3 / 2$, and $1 / 2$, respectively, in terms of a totally antisymmetric Lorentz tensor with Dirac spinor components. We point out that the exclusion of the spin-1/2 component by means of a subsidiary condition constructed by Fushchich and Nikitin 10 directly from the squared Pauli-Lubanski vector leads to an equation of motion for the spin-

\footnotetext{
The main problem with the relativization of the naive three-flavor quark model is that the Lorentz boost is responsible for transitions between the various $\mathrm{O}(3)$ multiplets and mixes different representations of the group $\mathrm{O}(3) \otimes \mathrm{SU}(6)$.
} 
$3 / 2$ resonance that avoids the main difficulties of the Rarita-Schwinger ansatz.

The paper is organized as follows. In the next two sections we briefly review for completeness the structure of nonstrange baryon spectra in terms of $\mathrm{O}(1,3) \otimes \mathrm{SU}(2)_{I}$ representations and present the classification of the $\Lambda$ hyperon excitations with special emphasis on the parity doublet patterns. In section IV we outline the construction of 'resonance cluster' propagators and present the new formalism for spin- $3 / 2$ fields. The paper closes with a short summary.

\section{Clustering of Baryon Spectra}

As long as the $\{j, j\}$ multiplets emerge in the $\mathrm{O}(4)$ symmetric Coulomb problem, 11 where they correspond to a principal quantum number $n=2 j+1$, the excitations of each single baryon isomultiplet with a $\pi N$ decay mode described in Ref. 3 appear amazingly patterned after the spectrum of the hydrogen atom with even principal quantum numbers $n=2,4,6$. In the $\mathrm{O}(3)$ reduction, the $\{j, j\}$ multiplets appear filled with states of (integer) angular momentum $l$ taking the values $l=0, \ldots, n-$ 1. All angular momenta contained in such representations have either natural or unnatural parity. Coupling a Dirac spinor to $l$ is standard and leads to states with total spin $\vec{J}=\vec{l} \otimes \frac{\overrightarrow{1}}{2}$. For $\mathrm{n}=2$ one finds, therefore, $J=\frac{1}{2}, \frac{1}{2}$, and $\frac{3}{2}$. ${ }^{\mathrm{b}}$ The parity of the latter states is exclusively determined by that of the underlying $\mathrm{O}(3)$ states. In case of natural parities, i.e., $0^{+}, 1^{-}$, one finds the sequence $J=\frac{1}{2}^{+}, \frac{1}{2}^{-}$, and $\frac{3}{2}^{-}$, while for unnatural parities, i.e., $0^{-}, 1^{+}$, one has $J=\frac{1}{2}^{-}, \frac{1}{2}^{+}$, and $\frac{3}{2}^{+}$, correspondingly. From this simple example it is seen that the parity of the state with the maximal spin allows one to reconstruct the parity (natural or unnatural) of the members of the Coulomb multiplet. Now, in considering the lowest excitations of the nucleon, the $\Lambda$, and the $\Delta$ resonances, one always finds the states $\mathrm{P}_{2 I, 1}, \mathrm{~S}_{2 I, 1}$, and $\mathrm{D}_{2 I, 3}$. This means that the parity of the corresponding intrinsic orbital angular momenta $l$ is natural and that these baryons are built upon a $0^{+}$vacuum as spontaneously selected in the Nambu-Goldstone mode of chiral symmetry. 12

Now, in considering the isospin $-3 / 2$ Lorentz multiplet with $n=4$, one finds all the required seven $\Delta$ resonances with spins ranging from $\frac{1}{2}^{-}$to $\frac{7}{2}^{+}$to be concentrated in the tiny mass region between $1900 \mathrm{MeV}$ and $1950 \mathrm{MeV}$. The positive parity of the highest spin $F_{37}$ state means that it has an intrinsic orbital angular momentum $l=3^{+}$of unnatural parity. Therefore, the second $\Delta$ resonance cluster is built upon a $0^{-}$vacuum and here chiral symmetry is restored. Within the $\mathrm{O}(1,3) \otimes \mathrm{SU}(2)_{I}$ scheme, the $\Delta$ spectrum below $2 \mathrm{GeV}$ appears complete. The $\mathrm{F}_{37}$ state has to be paralleled in the nucleon sector by a still-missing $\mathrm{F}_{17}$ resonance with a mass around $1700 \mathrm{MeV}$. The second cluster of nucleon resonances is consequently also expected to be built upon a vacuum of unnatural parity, and it is there where chiral symmetry restoration for nucleons will take place.

The parity $(-1)^{L+1}$ of a single $\pi N$ resonance $L_{2 I, 2 J}$ in standard notation 13 is determined in the present classification by either $(-1)^{l}$ or $(-1)^{l+1}$, depending on whether the parity of the intrinsic orbital angular momentum is natural or unnatural. In the present notation, $L$ takes the values of either $L=|l-1|,(l+1)$ for natural, or $L=l$ for unnatural parities. 
Lorentz Multiplet Structure of Baryons ...

In comparing the $\mathrm{n}=6$ nucleon and $\Delta$ states, four more missing resonances are predicted. These are the $\mathrm{H}_{1,11}, \mathrm{P}_{31}, \mathrm{P}_{33}$, and $\mathrm{D}_{33}$ states with masses between 2200 and $2400 \mathrm{MeV}$. In summary, five new, still unobserved non-strange resonances have been predicted in Ref. 3

Now, the comparison between the $\mathrm{O}(1,3) \otimes \mathrm{SU}(2)_{I}$ multiplets and the Höhler clusters performed in Table 1 reveals their identity. For example, the $\Delta$ cluster at $1900 \mathrm{MeV}$ is in fact nothing else but the isospin-3/2 $\{3 / 2,3 / 2\} \otimes[\{1 / 2,0\} \oplus\{0,1 / 2\}]$ Lorentz multiplet. Höhler clusters are, therefore, Lorentz multiplets.

Table 1. Correspondence between Höhler clusters and Lorentz multiplets. The missing resonances predicted here have been labeled by ' $m s$ '. For other notations, see text.

\begin{tabular}{|c|c|c|}
\hline $\mathbf{L}_{2 \mathrm{I}, 2 \mathrm{~J}}$ States & Pole (MeV) & Lorentz Multiplet \\
\hline $\begin{array}{l}\mathrm{S}_{11}, \mathrm{P}_{11}, \mathrm{P}_{13}, \mathrm{D}_{13} \\
\mathrm{D}_{15}, \mathrm{~F}_{15}, \mathrm{~F}_{17}^{m s}\end{array}$ & $\begin{array}{l}(1665 \pm 25) \\
-(55 \pm 15) \mathrm{i}\end{array}$ & $\begin{array}{l}\left\{\frac{3}{2}, \frac{3}{2}\right\} \otimes\left[\left\{\frac{1}{2}, 0\right\} \oplus\left\{0, \frac{1}{2}\right\}\right] \\
\otimes\left\{\frac{1}{2}\right\}_{I} \\
(n=4)\end{array}$ \\
\hline $\begin{array}{l}\mathrm{S}_{11}, \mathrm{P}_{11}, \mathrm{P}_{13}, \mathrm{D}_{13} \\
\mathrm{D}_{15}, \mathrm{~F}_{15}, \mathrm{~F}_{17}, \mathrm{G}_{17} \\
\mathrm{G}_{19}, \mathrm{H}_{19}, \mathrm{H}_{1,11}^{m s}\end{array}$ & $\begin{array}{l}(2110 \pm 50) \\
-(180 \pm 50) \mathrm{i}\end{array}$ & $\begin{array}{l}\left\{\frac{5}{2}, \frac{5}{2}\right\} \otimes\left[\left\{\frac{1}{2}, 0\right\} \oplus\left\{0, \frac{1}{2}\right\}\right] \\
\otimes\left\{\frac{1}{2}\right\}_{I} \\
(\mathrm{n}=6)\end{array}$ \\
\hline $\begin{array}{l}\mathrm{S}_{31}, \mathrm{P}_{31}, \mathrm{P}_{33}, \mathrm{D}_{33} \\
\mathrm{D}_{35}, \mathrm{~F}_{35}, \mathrm{~F}_{37}\end{array}$ & $\begin{array}{l}(1820 \pm 30) \\
-(120 \pm 30) \mathrm{i}\end{array}$ & $\begin{array}{l}\left\{\frac{3}{2}, \frac{3}{2}\right\} \otimes\left[\left\{\frac{1}{2}, 0\right\} \oplus\left\{0, \frac{1}{2}\right\}\right] \\
\otimes\left\{\frac{3}{2}\right\}_{I} \\
(\mathrm{n}=4)\end{array}$ \\
\hline $\begin{array}{l}\mathrm{S}_{31}, \mathrm{P}_{31}^{m s}, \mathrm{P}_{33}^{m s}, \mathrm{D}_{33}^{m s} \\
\mathrm{D}_{35}, \mathrm{~F}_{35}, \mathrm{~F}_{37}, \mathrm{G}_{37} \\
\mathrm{G}_{39}, \mathrm{H}_{39}, \mathrm{H}_{3,11}\end{array}$ & less established & $\begin{array}{l}\left\{\frac{5}{2}, \frac{5}{2}\right\} \otimes\left[\left\{\frac{1}{2}, 0\right\} \oplus\left\{0, \frac{1}{2}\right\}\right] \\
\otimes\left\{\frac{3}{2}\right\}_{I} \\
(\mathrm{n}=6)\end{array}$ \\
\hline $\begin{array}{l}\mathrm{S}_{01}(1800), \mathrm{P}_{01}(1810) \\
\mathrm{D}_{05}(1830), \mathrm{F}_{05}(1820) \\
\mathrm{P}_{03}(1890), \mathrm{D}_{03}^{m s}(2000 ?) \\
\mathrm{G}_{07}(2100), \mathrm{F}_{07}(2020)\end{array}$ & & $\begin{array}{l}\left\{0^{ \pm}\right\} \otimes\left[\left\{\frac{1}{2}, 0\right\} \oplus\left\{0, \frac{1}{2}\right\}\right] \\
\left\{0^{ \pm}\right\} \otimes\left[\left\{\frac{5}{2}, 0\right\} \oplus\left\{0, \frac{5}{2}\right\}\right] \\
\left\{0^{ \pm}\right\} \otimes\left[\left\{\frac{3}{2}, 0\right\} \oplus\left\{0, \frac{3}{2}\right\}\right] \\
\left\{0^{ \pm}\right\} \otimes\left[\left\{\frac{7}{2}, 0\right\} \oplus\left\{0, \frac{7}{2}\right\}\right]\end{array}$ \\
\hline
\end{tabular}

\section{Parity Doublets in the $\Lambda$ Hyperon Spectrum}

In Ref. 3 it was shown that none of the nonstrange resonances is exact parity duplicated. Indeed, exact fermion parity doublets must originate from underlying equal intrinsic orbital angular momenta of opposite parities. All the observed nearly mass degenerate nonstrange baryons of equal spins and opposite parities have, however, intrinsic orbital angular momenta differing by one unit. For example, the states $\mathrm{N}^{*}(1675)$ and $\mathrm{N}^{*}(1680)$ with $J^{P}=\frac{5}{2}^{-}$, and $J^{P}=\frac{5}{2}^{+}$, respectively, do not really form a pair because they belong to one and the same Lorentz multiplet (that cor- 
responding to $\mathrm{n}=4$ ), and their intrinsic orbital angular momenta and parities are $l=2^{-}$and $l=3^{+}$, respectively. On the contrary, in the present section it is shown that excited hyperons at comparatively high energies clusterize into exact parity doublets.

In Ref. 3 only the lowest $\Lambda$ hyperon excitations were considered. There, the first excited $\mathrm{S}_{01}, \mathrm{P}_{01}$, and $\mathrm{D}_{03}$ states were organized into the isosinglet $\{1 / 2,1 / 2\} \otimes$ $[\{1 / 2,0\} \oplus\{0,1 / 2\}]$ representation. The splitting between these $\mathrm{S}_{01}$ and $\mathrm{D}_{03}$ resonances reaches $175 \mathrm{MeV}$, and is comparable to the gap of about $150 \mathrm{MeV}$ between their mass average and the $\mathrm{P}_{01}$ state. This observation indicates that a further reduction of the four dimensional Lorentz multiplet into the trivial and the 3-vector representations is useful:

$$
\left\{\frac{1}{2}, \frac{1}{2}\right\} \longrightarrow\{0\} \oplus[\{1,0\} \oplus\{0,1\}] .
$$

The first excited $\mathrm{P}_{01}$ state is now attached to

$$
\Lambda\left(1600 ; 1 / 2^{+}\right) \simeq\left\{0^{+}\right\} \otimes\left[\left\{\frac{1}{2}, 0\right\} \oplus\left\{0, \frac{1}{2}\right\}\right],
$$

while the first $\mathrm{S}_{01}$ and $\mathrm{D}_{03}$ states are combined into

$$
\Lambda\left(1405 ; \frac{1}{2}^{-}\right) \quad \text { and } \quad \Lambda\left(1520 ; \frac{3}{2}^{-}\right) \simeq[\{1,0\} \oplus\{0,1\}] \otimes\left[\left\{\frac{1}{2}, 0\right\} \oplus\left\{0, \frac{1}{2}\right\}\right] .
$$

The $\Lambda(1600)$ resonance can therefore be considered as an isolated second spin- $1 / 2^{+}$ $\Lambda$ hyperon that parallels the Roper resonance of the nucleon. From the last equation it is further seen that the first isosinglet spin- $1 / 2^{-}$resonance has an intrinsic angular momentum $1^{-}$and cannot be considered as the parity partner to $\Lambda(1116)$ of $0^{+}$ intrinsic angular momentum. The $\{1,0\} \oplus\{0,1\}$ pattern appears once more in the next resonance cluster including the $\Lambda\left(1670 ; 1 / 2^{-}\right)$and $\Lambda\left(1690 ; 3 / 2^{-}\right)$states. Here again, the second $S_{01}$ excitation is still not a parity partner to $\Lambda(1116)$. At 1800 $\mathrm{MeV}$, however, one immediately encounters well-pronounced parity doublet clusters according to

$$
\begin{aligned}
& \Lambda\left(1800 ; 1 / 2^{-}\right) \simeq\left\{0^{-}\right\} \otimes\left[\left\{\frac{1}{2}, 0\right\} \oplus\left\{0, \frac{1}{2}\right\}\right], \\
& \Lambda\left(1810 ; 1 / 2^{+}\right) \simeq\left\{0^{+}\right\} \otimes\left[\left\{\frac{1}{2}, 0\right\} \oplus\left\{0, \frac{1}{2}\right\}\right], \\
& \Lambda\left(1820 ; 5 / 2^{+}\right) \simeq\left\{0^{+}\right\} \otimes\left[\left\{\frac{5}{2}, 0\right\} \oplus\left\{0, \frac{5}{2}\right\}\right], \\
& \Lambda\left(1830 ; 5 / 2^{-}\right) \simeq\left\{0^{-}\right\} \otimes\left[\left\{\frac{5}{2}, 0\right\} \oplus\left\{0, \frac{5}{2}\right\}\right] .
\end{aligned}
$$

In other words, in the $\Lambda$ hyperon sector the parity duplication occurs for the first time at around $1800 \mathrm{MeV}$. A possible interpretation of this phenomenon is that at that scale the strange quark has already accumulated enough energy to move sufficiently far away from the nonstrange quarks, giving rise to a reflection-asymmetric 
nucleon shape. That such a shape is responsible for the occurrence of parity dgublets in baryon spectra was to our knowledge initiated by Dönau and Reinhardt. 14 Later on, extensive work in that direction was performed, e.g., by Iachello as well as by Bijker and Leviatan. 15 For the reasons given above, the $\Lambda$ hyperon spectrum above $1800 \mathrm{MeV}$ presents itself as patterned after the classification schemes of Refs. 14 and 15 rather than after the $\mathrm{O}(1,3) \otimes \mathrm{SU}(2)_{I}$ scheme. Therefore, for $\Lambda$ hyperons the restoration of chiral symmetry takes place above $1800 \mathrm{MeV}$. Similar patterns have been reported by Balachandran and Vaidya 16 in the spectra of heavy flavor $(c b u)$ and $(c b d)$ baryons. There, it was shown that once heavy flavor quarks participate in the baryon structure, the baryon shape becomes reflection-asymmetric, thus giving rise to parity doublet patterns.

\section{Propagating Two-Resonance Clusters and Isolated Spin-3/2 States}

To find the relativistic equation of motion for a given $\{j, j\}$ representation of the Lorentz group, one has to construct the corresponding projection operator. For that one has to find the $(2 j+1)(2 j+1)$ matrices $D^{j j}(b(\vec{p}))$ that carry the boost $b(\vec{p})$ from the rest frame to the system where the particle is moving with momentum $\vec{p}$ as well as the respective matrix of the three-space inversion $\mathcal{P}$. The projector onto $\{j, j\}$ is then expressed as:

$$
\Pi(p)^{j, j}=D^{j, j}(b(\vec{p})) \frac{1}{2}\left(\eta_{p} \mathcal{P}+1\right)\left(D^{j, j}(b(\vec{p}))^{-1} .\right.
$$

Here, $\eta_{p}$ is the parity of the vacuum where the $\{j, j\}$ multiplet is built upon. With that, the projector $\left(\Pi_{\mu \nu}^{R S}(p)\right)$ onto the 16 -dimensional RS-field $\{1 / 2,1 / 2\} \otimes$ $[\{1 / 2,0\} \oplus\{0,1 / 2\}]$ (subsequently denoted by $\Psi_{\mu}(p)$ ) is found as

$$
\Pi_{\mu \nu}^{R S}(p)=\frac{p^{\lambda} \gamma_{\lambda}+M}{2 M}\left(g_{\mu \nu}-\frac{p_{\mu} p_{\nu}}{M^{2}}\right)
$$

with $\gamma_{\lambda}$ being the standard Dirac matrices. The field $\Psi_{\mu}(p)$ represents a Lorentz vector with Dirac spinor components. From that, the corresponding relativistic equation of motion is obtained as

$$
\Pi_{\mu \nu}^{R S}(p) \Psi^{\nu}(p)=\Psi_{\mu}(p) .
$$

Note that a polar vector $\Psi(p)_{\mu}$ collects the $\mathrm{S}_{2 I, 1}, \mathrm{P}_{2 I, 1}$, and $\mathrm{D}_{2 I, 3}$ resonances, while an axial one unites the $\mathrm{P}_{2 I, 1}, \mathrm{~S}_{2 I, 1}$, and $\mathrm{P}_{2 I, 3}$ states, respectively. This shows that the redundant components of the RS field are not, as often said, unphysical ones. Equation (7) is automatically satisfied if the Dirac equation is valid for each component of the field $\Psi_{\mu}$, on the one side,

$$
\frac{1}{2 M}\left(p^{\lambda} \gamma_{\lambda}+M\right) \Psi_{\mu}(p)=\Psi_{\mu}(p),
$$

and if $\Psi_{\mu}$ satisfies

$$
\left(g^{\mu \nu}-\frac{p^{\mu} p^{\nu}}{M^{2}}\right) \Psi_{\nu}(p)=\Psi^{\mu}(p),
$$


on the other side. Equation (9) is equivalent to Proca's equation $p^{\mu} \Psi_{\mu}(p)=0$ and is usually considered as a supplementary condition to Eq. (8). It eliminates one of the four degrees of freedom of the Lorentz vector $\Psi_{\mu}$ (which can be considered to be the time component) and ensures mapping of the selfconjugate Lorentz multiplet $\{1 / 2,1 / 2\}$ onto the bi-vector $\{1,0\} \oplus\{0,1\}$. For concreteness, we here consider the case of a polar Lorentz vector. In that case it is the $\mathrm{P}_{2 I, 1}$ resonance that is removed by means of Proca's equation. The remaining 12 components unite the $\mathrm{S}_{2 I, 1}$ and $\mathrm{D}_{2 I, 3}$ states. Therefore, the covariant object,

$$
\mathcal{S}_{\mu \nu}(p)=\frac{\Pi_{\mu \nu}^{R S}(p)}{p^{2}-M^{2}}=\frac{\left(p^{\lambda} \gamma_{\lambda}+M\right)\left(g_{\mu \nu}-\frac{p_{\mu} p_{\nu}}{M^{2}}\right)}{2 M\left(p^{2}-M^{2}\right)},
$$

describes the propagator of the cluster state containing the resonances $\mathrm{D}_{2 I, 3}$ and $\mathrm{S}_{2 I, 1}$ considered as mass degenerate. This is quite a reasonable approximation as the splitting between these states is only $15 \mathrm{MeV}$ for the isodoublet, and only 50 $\mathrm{MeV}$ for the isoquadruplet. The $\mathrm{P}_{2 I, 1}$ resonance can be considered separately by means of the Dirac equation. This way, the problem of the mass splitting within the $\{1 / 2,1 / 2\}$ multiplets is solved satisfactorily.

The scheme outlined above can be extended to multi-resonance cluster states. However, the description of isolated higher-spin $\{J, 0\} \oplus\{0, J\}$ states like the two lowest $\Delta$ resonances at $1232 \mathrm{MeV}$ and $1600 \mathrm{MeV}$, respectively, or the parity duplicated higher-spin states observed in the $\Lambda$ hyperon spectrum, still remains problematic. Recall that the final isolation of the spin-3/2 component from Eqs. (8) and (9) is performed by means of a second subsidiary condition on $\Psi_{\mu}(p)$, taken ad

$$
\gamma^{\mu} \Psi_{\mu}(p)=0
$$

In doing this, the difficulty arises that the subsidiary conditions, in particular Eq. (9), are fulfilled only on-mass shell, so that off-mass shell the separation between the spin- $1 / 2$ and the spin- $3 / 2$ fields is no longer guaranteed.17 This shows up in the appearance of arbitrary off-shell parameters both in the $\Delta$ propagator and the $N \Delta \gamma$ vertex

An alternative linear relativistic equation of motion for spin $-3 / 2$ fields can be obtained in coupling a Dirac spinor directly to a 6 -dimensional bi-vector rather than to a $4-$ vector:

$$
\Psi_{a} \simeq[\{1,0\} \oplus\{0,1\}] \otimes\left[\left\{\frac{1}{2}, 0\right\} \oplus\left\{0, \frac{1}{2}\right\}\right], \quad a=1, \ldots, 6 .
$$

In the following, Latin indices will be used to label the bi-vector components. This 24-dimensional representation, which has been mapped onto a bi-vector with Dirac spinor components, describes a doubled two-resonance spin-3/2 and spin$1 / 2$ cluster together with the corresponding anti-cluster. The doubling accounts for covering of space-time reflection operations. Equation (12) was originally proposed by Fushchich and Nikitin (FN).10 Here we will separate the Dirac from the bivector indices rather than operate with the complete 24 dimensional FN field as 
Lorentz Multiplet Structure of Baryons...

was done in the original paper.10 The advantage of our scheme will become clear in constructing interaction Lagrangians. Now, each component of $\Psi_{a}$ satisfies the Dirac equation,

$$
(\not p-M) \Psi_{a}(p)=0, \quad a=1, \ldots, 6,
$$

with $\not p:=p^{\mu} \gamma_{\mu}$. In order to obtain a description of the spin $-3 / 2$ field alone, a covariant subsidiary condition has to be imposed on the wave function $\Psi_{a}(p)$ that eliminates the spin $-1 / 2$ component. This subsidiary condition can be constructed directly from the Pauli-Lubanski vector,18

$$
\left(W_{\mu}\right)_{a b}=-\frac{1}{2} \epsilon_{\mu \nu \rho \tau}\left(S^{\nu \rho}\right)_{a b} p^{\tau}, \quad a, b=1, \ldots, 6,
$$

where $\left(S_{\nu \rho}\right)_{a b}$ are the spin-matrices for the composite bi-vector-Dirac spinor representation

$$
\left(S_{\nu \rho}\right)_{a b}=\frac{1}{2} \sigma_{\nu \rho}\left(1_{6}\right)_{a b}+\left(\sigma_{\nu \rho}^{(1)}\right)_{a b} 1_{4} .
$$

Here, $\sigma_{\mu \nu}=i\left[\gamma_{\mu}, \gamma_{\nu}\right] / 2$ and $\sigma_{\mu \nu}^{(1)}=i\left[\gamma_{\mu}^{(1)}, \gamma_{\nu}^{(1)}\right] / 4$, respectively, where the $6 \times 6$ unit matrix acts onto the bi-vector, while the $4 \times 4$ unit matrix acts within the Dirac spinor space. The matrices $\gamma_{\mu}^{(1)}$ are introduced as

$$
\gamma_{0}^{(1)}=\left(\begin{array}{lr}
0_{3} & -1_{3} \\
-1_{3} & 0_{3}
\end{array}\right)
$$

where $1_{3}$ and $0_{3}$ denote in turn the three-dimensional unit and zero matrices, while the remaining 6 -dimensional spin- $1 \vec{\gamma}^{(1)}$ matrices are defined as

$$
\vec{\gamma}^{(1)}=2\left(\begin{array}{cc}
0_{3} & \vec{S} \\
-\vec{S} & 0_{3}
\end{array}\right)
$$

with

$$
\left(S_{\alpha}\right)_{\beta \eta}=-i \epsilon_{\alpha \beta \eta}, \quad \alpha, \beta, \eta=1,2,3 .
$$

At rest the eigenvectors to $S_{3}$ are given by

$$
\vec{\epsilon}_{1}=\frac{1}{\sqrt{2}}\left(\begin{array}{c}
1 \\
i \\
0
\end{array}\right), \quad \vec{\epsilon}_{2}=\frac{1}{\sqrt{2}}\left(\begin{array}{c}
1 \\
-i \\
0
\end{array}\right), \quad \vec{\epsilon}_{3}=\left(\begin{array}{l}
0 \\
0 \\
1
\end{array}\right) .
$$

Note that the $\gamma_{\mu}^{(1)}$ matrices do not constitute a Clifford algebra, but they possess well defined anti-commutators as the three matrices $S_{\alpha}$ from Eq. (18) satisfy the $\mathrm{su}(2)$ algebra. Finally, we wish to introduce the notation for the bi-vector-Dirac spinor as

$$
\Psi=\left(\begin{array}{l}
\vec{\phi} \\
i \vec{\chi}
\end{array}\right)
$$

where $\vec{\phi}$ and $\vec{\chi}$ are three-dimensional vectors with Dirac spinor components. At rest and within a basis where the index $a$ counts the angular momentum states $J M$ one 
finds

$$
\begin{aligned}
\phi_{J M}= & \sum_{\substack{m=0, \pm 1 \\
s_{3}= \pm 1 / 2}}\left(1 m_{1} \frac{1}{2} s_{3} \mid J M\right) \epsilon_{1 m} u_{s}, \\
u_{\frac{1}{2}}=\left(\begin{array}{l}
1 \\
0
\end{array}\right), \quad & u_{-\frac{1}{2}}=\left(\begin{array}{c}
0 \\
1
\end{array}\right) .
\end{aligned}
$$

Now, the squared Pauli-Lubanski vector is calculated as

$$
\left(W_{\mu} W^{\mu}\right)_{a b}=\frac{1}{2}\left(S_{\mu \nu}\right)_{a b^{\prime}}\left(S^{\mu \nu}\right)_{b^{\prime} b} p_{\lambda} p^{\lambda}-\left(S_{\mu \nu}\right)_{a b^{\prime}}\left(S^{\sigma \nu}\right)_{b^{\prime} b} p^{\mu} p_{\sigma} .
$$

With that the subsidiary condition to Eq. (13), which eliminates the redundant spin- $-1 / 2$ component can be deduced as

$$
\left(W^{\mu} W_{\mu}\right)_{a b} \Psi_{b}^{s}(p)=-p^{2} s(s+1) \Psi_{a}^{s}(p), \quad s=\frac{3}{2} .
$$

For $p^{2} \neq 0$ Eq. (24) is equivalently cast into the form

$$
\begin{aligned}
P_{a b}^{s}(p) \Psi_{b}^{s}(p) & =\Psi_{a}^{s}(p) \\
P_{a b}^{s}(p) & =-\frac{1}{2 s}\left[\frac{1}{p^{2}}\left(W^{\mu} W_{\mu}\right)_{a b}+s(s-1) \delta_{a b}\right], \quad s=\frac{3}{2},
\end{aligned}
$$

where the projection operator $P_{a b}^{s}(p)$ onto the subspace with the maximal spin $s$ has been introduced. It turns out to be quite favorable to consider Eq. (25) as the equation of motion while Eq. (13) is considered as the subsidiary condition. The latter is simply incorporated into Eq. (25) in rewriting $P^{s}(p)$ to the equivalent form 10

$$
\begin{aligned}
P_{a b}^{s}(p) \Psi_{b}^{s}(p)= & \left(\frac{1+\gamma_{5}}{2}+\frac{1-\gamma_{5}}{2} \frac{\not p}{M}\right) P_{a b}^{s}(p) \Psi_{b}^{s}(p) \\
= & \frac{\not p+M}{2 M}\left(1+\gamma_{5}\right)(-1) \frac{1}{4 s}\left[\left(S^{\mu \nu} S_{\mu \nu}\right)_{a b}+2 s(s-1) \delta_{a b}\right. \\
& \left.-2\left(S_{\mu \nu} S^{\sigma \nu}\right)_{a b} \frac{p^{\mu} p_{\sigma}}{p^{2}}\right] \frac{\not p}{M} \Psi_{b}^{s}(p), \\
P^{s}(p)^{2}=P^{s}(p), \quad P^{s-1}(p)= & 1-P^{s}(p), \quad P^{s}(p) P^{s-1}(p)=0, \quad s=\frac{3}{2},
\end{aligned}
$$

where $\gamma_{5}=i \gamma_{0} \gamma_{1} \gamma_{2} \gamma_{3}$, and use of Eqs. (23) and (24) was made. From Eq. (26) the propagator of the spin $-3 / 2$ field is deduced as the following matrix:

$$
\begin{aligned}
\mathcal{S}^{\frac{3}{2}}(p) & =\frac{P^{\frac{3}{2}}(p)}{p^{2}-M^{2}} \\
S^{\frac{3}{2}}(p) & =-\frac{(\not p+M)\left(1+\gamma_{5}\right)\left[\left(S^{\mu \nu} S_{\mu \nu}+\frac{3}{2}-\frac{2}{p^{2}} S_{\mu \nu} S^{\sigma \nu} p^{\mu} p_{\sigma}\right] \not p\right.}{12 M^{2}\left(p^{2}-M^{2}\right)}
\end{aligned}
$$


It is easily verified that Eq. (26) is obtained from the following Lagrangian

$$
\begin{aligned}
\mathcal{L}= & \bar{\Psi}_{a}^{s}(\not p+M)\left(1+\gamma_{5}\right)\left[\left(S^{\mu \nu} S_{\mu \nu}\right)_{a c}+2 s(s-1) \delta_{a c}\right. \\
& \left.-2\left(S_{\mu \nu} S^{\sigma \nu}\right)_{a c} \frac{p^{\mu} p_{\sigma}}{M^{2}}\right] \frac{\not p}{M} \Psi_{c}^{s}+8 M s \bar{\Psi}_{d}^{s} \Psi_{d}^{s}, \quad s=\frac{3}{2},
\end{aligned}
$$

where $p^{2}=M^{2}$ has been set. The introduction of a minimal coupling of the field $\Psi$ to the photon via $p_{\mu} \rightarrow p_{\mu}-e A_{\mu}$ preserves the form (and therefore the compatibility) of both the Eqs. (13) and (26). As long as Eq. (25) is not restricted to on-mass shell only, the separation of the spin- $3 / 2$ and spin $-1 / 2$ components does not cause any more problems. The interacting propagator is now obtained from Eq. (27) by replacing $\not p$ through $\hbar:=\left(p^{\mu}-e A^{\mu}\right) \gamma_{\mu}$. In 10 the frame independence of the eigenvalue of the Pauli-Lubanski vector was exploited to replace the second term of Eq. (23) by its equivalent in the rest frame, $-S^{\mu \nu} S_{\mu \nu} / 4$. For that case, the proof was given, that the spin- $3 / 2$ particle does not any longer move with superluminal velocity in an external magnetic field.

Now, in mapping the bi-vector onto a totally antisymmetric field tensor $V_{[\mu \nu]}$ according to

$$
\begin{aligned}
V_{0 \alpha} & =\phi_{\alpha}, \quad \alpha=1,2,3, \\
V_{12}=\chi_{3}, \quad V_{23} & =\chi_{1}, \quad V_{31}=\chi_{2},
\end{aligned}
$$

one can map the 24-dimensional representation $\Psi_{a}(p)$ onto a 2nd rank antisymmetric Lorentz tensor with Dirac spinor components according to

$$
\Psi_{a}(p) \simeq \psi_{[\mu \nu]}(p),
$$

where the brackets '[ ]' have been used to emphasize the antisymmetrization. The favor of our notation over that of the original paper 10 is the that the spin- $3 / 2$ field is described as a fully covariant object. Now the $N D_{2 I, 3} \gamma$ vertex in coordinate space can be constructed as

$$
\mathcal{L}_{N D_{2 I, 3} \gamma}=g \bar{\psi}_{\mu \nu} \psi_{N} F^{\mu \nu}+g_{1} \bar{\psi}^{\mu \nu} \sigma_{\mu \lambda} \sigma_{\nu \eta} \psi_{N} F^{\lambda \eta}
$$

where $\psi_{N}$ stands for the Dirac spinor of the nucleon, $F^{\mu \nu}$ is the electromagnetic field strength tensor, while $g$ and $g_{1}$ are the coupling strengths. Other couplings as

$$
\bar{\psi}^{\mu \nu} \sigma_{\mu \nu} \sigma_{\rho \tau} \psi_{N} F^{\rho \tau} \text {, }
$$

will not contribute as $\sigma_{\mu \nu} \psi^{\mu \nu}$ vanishes. Indeed, at rest, this condition reduces to $\vec{\sigma} \cdot \vec{\phi}=0$ and ensures validity of Eq. (25) in the rest frame, where it takes the form

$$
\left(\vec{S}+\frac{1}{2} \vec{\sigma}\right)^{2} \vec{\phi}=\frac{15}{4} \vec{\phi}
$$

As long as Eq. (25) is valid off-mass shell too, the vertex constructed in Eq. (31) does not contain any off-shell parameters. The $N D_{3 I, 3} \pi$ vertex in coordinate space can 
be constructed in analogy to Eq. (31) by replacing $F_{\mu \nu}$ by $U_{\mu \nu}=\left[U^{\dagger} \partial_{\mu} U, U^{\dagger} \partial_{\nu} U\right]$ with $U$ being the well known dimensionless quaternion chiral meson field $U=$ $(\sigma+i \vec{\pi} \cdot \vec{\tau}) / f_{\pi}$ underlying the Skyrme model.

\section{Summary}

In the present paper it was shown that the clustering in baryon spectra pointed out by Höhler is well interpreted in terms of multiplets of the Lorentz-isospin group $\mathrm{O}(1,3) \otimes \mathrm{SU}(2)_{I}$. For nonstrange baryons these clusters are of the type $\{j, j\} \otimes$ $[\{1 / 2,0\} \oplus\{0,1 / 2\}] \otimes\{I\}$ with $n=2 j+1$ even. For the $\Lambda$ hyperons with masses below $1800 \mathrm{MeV}$ the Lorentz clusters have been shown to be of the type $\{1,0\} \oplus$ $\{0,1\} \otimes[\{1 / 2,0\} \oplus\{0,1 / 2\}]$ while above $1800 \mathrm{MeV}$ they are parity-duplicated $\{J, 0\} \oplus$ $\{0, J\}$ Weinberg-Ahluwalia states. The suggestion was made that in evaluating Feynman graphs in such processes as meson production off protons, the propagation of resonance clusters as intermediate states might be more appropriate and easier to handle as compared to the propagation of isolated higher-spin states. Clusters corresponding to Lorentz multiplets with $n=2 j+1$ odd have not been observed so far. At the present stage it is still not clear if their existence is forbidden for some dynamical reasons or if they have to be viewed as missing clusters because of the property to decouple from the $\pi N$ decay channel. The possibility to observe missing resonances in other than the $\pi N$ decay channel was discussed in a recent work by Capstick and Roberts.19

In addition, for nonstrange baryons the Nambu-Goldstone mode of chiral symmetry was shown to extend only to the first excited $\mathrm{P}_{2 I, 1}, \mathrm{~S}_{2 I, 1}$, and $\mathrm{D}_{2 I, 3}$ states (with $I=1 / 2$, and $I=3 / 2$ ). The next higher Lorentz multiplets with $n=2 j+1>2$ were shown to be built upon a $0^{-}$vacuum and hence, chiral symmetry is restored there. For $\Lambda$ hyperons chiral symmetry is restored above $1800 \mathrm{MeV}$.

As a promising candidate for describing the quark dynamics underlying the $\mathrm{O}(1,3) \otimes \mathrm{SU}(2)_{I}$ spectrum-generating algebra of Ref. 3 one may consider the covariant quark-diquark model based on solving the Bethe-Salpeter equation in the rapidly converging $\mathrm{O}(4)$ basis of the Gegenbauer polynomials as outlined in.20

Finally, it was demonstrated that the description of spin- $3 / 2$ particles in terms of a 2 nd rank antisymmetric Lorentz tensor with Dirac spinor components does not contain any off-shell parameters and avoids the main difficulties of the description based upon a 4 -vector with Dirac spinor components.

\section{Acknowledgements}

Valuable correspondence with Anatoly Nikitin is gratefully acknowledged. This work was supported by the Deutsche Forschungsgemeinschaft (SFB 201).

\section{References}

1. G. Höhler and H. M. Staudenmaier, in $\pi N$ Newsletter, Proceedings of the Sixth International Symposium on Meson-Nucleon Physics and the Structure of the Nucleon, 
ed. D. Drechsel et al. 11, 7 (1995); ibid. 194; G. Höhler, in Pion-Nucleon Scattering Landolt-Börnstein Vol. I/9b2, ed. H. Schopper (Springer, 1983).

2. B. M. K. Nefkens, in $\pi N$ Newsletter, Proceedings of the Sixth International Symposium on Meson-Nucleon Physics and the Structure of the Nucleon, ed. D. Drechsel et al. 11, 145 (1995).

3. M. Kirchbach, Mod. Phys. Lett. A12, 2373 (1997).

4. S. Weinberg, Phys. Rev. 133, B1318 (1964); ibid. 134, B852 (1965); ibid. 138, B988 (1965), Sec. IId; S. Weinberg, in Brandeis University Summer Institute in Theoretical Physics: Lectures on Fields and Particles, Vol. II, ed. S. Deser and K. W. Ford (Prentice Hall, 1965).

5. K. Johnson and E. C. G. Sudarshan, Ann. Phys. 13, 126 (1961).

6. G. Velo and D. Zwanziger, Phys. Rev. 186, 1337 (1969); W. Tsai , Phys. Rev. D4, 3643 (1971); T. Goldman and W. Tsai, ibid., 3648.

7. M. Bennmerrouche, R. M. Davidson, and N. C. Mukhopadhyay, Phys. Rev. C39, 2339 (1989).

8. D. V. Ahluwalia, Ph. D. thesis, Texas A\&M University, 1991, Dissertation Abstracts International B52, 4792-B (1992); D. V. Ahluwalia, Int. J. Mod. Phys. A11, 1855 (1996); D. V. Ahluwalia, in The Present Status of the Quantum Theory of Light, ed. S. Jeffers et al., p. 443 (Kluwer Academic Publishers, 1997); D. V. Ahluwalia and D. J. Ernst, Int. J. Mod. Phys. E2, 397 (1993).

9. W. Rarita and J. Schwinger, Phys. Rev. 60, 61 (1941); D. Lurie, Particles and Fields (John Wiley \& Sons, 1968).

10. V. I. Fushchich and A. G. Nikitin, Sov. J. Part. Nucl. 9, 205 (1978); V. I. Fushchich and A. G. Nikitin, Symmetries of equations of quantum mechanics (Allerton Press Inc., NY, 1994).

11. J. D. Elliot and P. G. Dawber, Symmetries in Physics (Macmillan, 1979).

12. S. Coleman, in Aspects of Symmetry, Selected Erice Lectures (Cambridge University Press, 1985).

13. Particle Data Group, R. M. Barett et al., Phys. Rev. D54, 1 (1996).

14. F. Dönau and H. Reinhardt, Spectroscopy of Rotating Quark Bags, preprint NBI-84-46 (1984), unpublished.

15. F. Iachello, Phys. Rev. Lett. 62, 2440 (1989); R. Bijker and A. Leviatan, nucl-th/9701037.

16. A. P. Balachandran and S. Vaidya, Phys. Rev. Lett. 78, 13 (1997).

17. P. Moldauer and K. M. Case, Phys. Rev. 102, 279 (1956).

18. C. Itzykson and J. -B. Zuber, Quantum Field Theory (McGraw-Hill, 4th printing, 1988).

19. S. Capstick and W. Roberts, nucl-th/9708048

20. K. Kusaka, G. Piller, A. W. Thomas, and A. G. Williams, Phys. Rev. D55, 5299 (1997). 